\title{
Microglial activation and neurobiological alterations in experimental autoimmune prostatitis-induced depressive-like behavior in mice
}

This article was published in the following Dove Press journal: Neuropsychiatric Disease and Treatment

\begin{abstract}
He-Xi Du, ${ }^{1-3}$ Xian-Guo Chen, ${ }^{1-3}$ Li Zhang, ${ }^{1-3}$ Yi Liu, ${ }^{\text {I-3 Chang- }}$ Sheng Zhan, ${ }^{\mathrm{I}-3}$ Jing Chen, ${ }^{\mathrm{I}-3}$ Yong Zhang, ${ }^{\text {I-3 }}$ Zi-Qiang Yu, ${ }^{\text {I-3 }}$ Jin Zhang, ${ }^{4,5}$ Hong-Yi Yang, ${ }^{4,5}$ Kai Zhong, ${ }^{4,5}$ Chao-Zhao Liang ${ }^{1-3}$

'Department of Urology, The First Affiliated Hospital of Anhui Medical University, Anhui Medical University, Hefei 230022, People's Republic of China; ${ }^{2}$ Institute of Urology, Anhui Medical University, Hefei 230022,

People's Republic of China; ${ }^{3}$ Anhui Province Key Laboratory of Genitourinary Diseases, Anhui Medical University, Hefei 230022, People's Republic of China; ${ }^{4}$ High Magnetic Field Laboratory, Hefei Institutes of Physical Science, Chinese Academy of Sciences, Hefei 23003I, People's Republic of China;

${ }^{5}$ Key Laboratory of Anhui Province for High Magnetic Resonance Imaging, Hefei 23003I, People's Republic of China
\end{abstract}

Correspondence: Kai Zhong High Magnetic Field Laboratory, Hefei Institutes of Physical Science, Chinese Academy of Sciences, No. 350 Shushan Lake Road, Hefei, People's Republic of China

Tel +86 I 36755 I 5810

Fax +865516559 1647

Email kzhong@hmfl.ac.cn

Chao-Zhao Liang

Department of Urology, The First

Affiliated Hospital of Anhui Medical

University, Anhui Medical University, No.

218 Jixi Road, Hefei, People's Republic of China

Tel +86 I 3505604595

Fax +8655163633742

Email ayfymw@163.com
Background: Patients with chronic prostatitis/chronic pelvic pain syndrome (CP/CPPS) frequently show depressive symptoms clinically and increasing evidence indicates a correlation between CP/CPPS and depression. However, the underlying mechanisms of CP/CPPSrelated depression remain poorly understood. Here, we sought to determine the role of hippocampal microglial activation and neurobiological changes in a mouse model of experimental autoimmune prostatitis (EAP)-induced depression and the treatment efficacy of Chinese herb extract baicalein.

Methods: EAP was induced through intradermal injection of prostate antigen and adjuvant twice. Then, mice were assessed for affective behaviors in the open field test, elevated plus maze, forced swim test, and tail suspension test. The morphology and function of microglia and astrocytes were detected by immunofluorescence, Western blotting, and transmission electron microscopy. Proinflammatory mediators along with serotonin transporter (SLC6A4/ SERT) and indoleamine 2,3-dioxygenase (IDO) were quantified with reverse transcriptionpolymerase chain reaction (RT-PCR), and serum serotonin concentrations were measured by enzyme-linked immunosorbent assay (ELISA). Proton magnetic resonance spectroscopy $\left({ }^{1} \mathrm{H}-\right.$ MRS) was performed to measure hippocampal glutamate levels. In addition, baicalein was used in a subset of EAP mice to test its anti-depressant action.

Results: EAP was successfully established and induced depressive- and anxiety-like behavior in mice. Increasing levels of co-expressed ionized calcium-binding adapter molecule 1 (Iba1) and glial fibrillary acidic protein (GFAP) and ultrastructural observations suggested microglial activation and reactive astrocytosis in the hippocampus. These activated microglia resulted in increased expressions of multiple proinflammatory cytokines. Simultaneously, EAP mice showed higher gene expressions of SLC6A4 and IDO and lower concentrations of serotonin. ${ }^{1} \mathrm{H}$-MRS indicated a decrease in the glutamate + glutamine $(\mathrm{Glx}) /$ total creatine $(\mathrm{tCr})$ ratio in EAP mice. Furthermore, baicalein treatment alleviated the depressive-like behavior and neuroinflammation by suppressing the nuclear factor-kappa $\mathrm{B}(\mathrm{NF}-\kappa \mathrm{B})$ pathway.

Conclusion: Our data indicate that EAP-induced depressive-like behavior is linked to microglia activation and subsequent neurotransmitter metabolism. Moreover, baicalein attenuates behavioral changes by inhibiting neuroinflammation via downregulation of the NF- $\mathrm{kB}$ pathway.

Keywords: EAP, microglial activation, serotonin, neurochemical changes, baicalein

\section{Introduction}

Prostatitis is a common disorder in the male population with an estimated prevalence ratio between $2.7 \%$ and $9.7 \%$ worldwide. ${ }^{1}$ According to the National Institutes of Health (NIH) classification, prostatitis is categorized into four types; 
category III is also called chronic prostatitis/chronic pelvic pain syndrome (CP/CPPS), accounting for the vast majority of all prostatitis diagnoses. ${ }^{2}$ The most common clinical presentations of $\mathrm{CP} / \mathrm{CPPS}$ are ongoing pelvic pain with urinary tract symptoms or sexual dysfunction. Besides these symptoms, patients with CP/CPPS are more likely to experience depressive symptoms than general male population, which seriously interferes with their quality of life (QOL). ${ }^{3,4}$ According to the literatures, depression can be found in as high as $78 \%$ of CP/CPPS patients. ${ }^{5}$ Nonetheless, to date, the molecular and cellular mechanism behind CP/CPPS and depression remains obscure.

In recent years, the crucial role of microglial activation in the development of depression has drawn increasingly more attention. ${ }^{6-8}$ Microglia, the resident macrophage of the central nervous system (CNS), is regarded as the major player in mediating brain immunological function. It responds to multiple pathological conditions such as inflammation process and brain damage and becomes an activated phenotype by enlarging its cell body and retracting processes. ${ }^{9}$ The activated microglia can cause the overproduction of several pro-inflammatory mediators, such as TNF- $\alpha$ and IL-1 $\beta$, which contribute to the formation of depression. ${ }^{6,10,11}$ Intriguingly, previous researches in addressing the CP/CPPS-related mental health disorders uncovered clues that prostate-derived proinflammatory factors can cross the blood brain barrier and reach several brain areas. ${ }^{12}$ At this level, these cytokines may further activate microglial cells. ${ }^{13}$ In light of the aforementioned data, it is reasonable to hypothesize that autoimmunitydriven prostatitis leads to the formation of depression by eliciting microglial activation in the CNS.

In fact, neurochemical transmitter system abnormalities are assumed to be a potential pathology in depression. ${ }^{14}$ Serotonin (5-hydroxytryptamine, 5-HT) and glutamate are two important neurotransmitters that exert an impact on mood. Compelling evidence has shown that proinflammatory cytokines from activated microglia can enhance the gene expression of indoleamine 2,3 dioxygenase (IDO), which reflects the catabolism of tryptophan and ultimately causes the loss of 5-HT. ${ }^{15,16}$ Also, central inflammatory activation is involved in the regulation of the serotonin transporter (SLC6A4/SERT). ${ }^{17}$ Moreover, microgliamediated inflammatory response can lead to alterations of glutamate metabolism by influencing the function of astrocyte. ${ }^{18}$ Based on these findings, we postulated that microglial-dependent changes in the serotonergic and glutamatergic system may be responsible for depressive behavior in CP/CPPS patients.

Baicalein (5,6,7-trihydroxyflavone) is a natural flavonoid isolated from the Chinese herb Scutellaria baicalensis. It exhibits several notable biological activities, such as antiinflammatory, anti-fibrogenic, and antitumor activities. ${ }^{19-21}$ Moreover, baicalein can promote neuronal and behavioral recovery via suppressing neuroinflammation. ${ }^{22}$ Baicalein also has a potential therapeutic effect on treating depression via inactivating the NF- $\mathrm{KB}$ signaling pathway. ${ }^{23}$ Despite this finding, whether baicalein has a beneficial effect on ameliorating EAP-induced depressive-like behavior in mice remains undetermined.

In this work, we aim to investigate the role of microglial activation and its involvement in hippocampal neurobiological alterations in a mouse model of experimental autoimmune prostatitis (EAP)-induced depression. Our data indicated that microglial activation very likely plays a central role in mediating depressive-like behavior in EAP mice. Moreover, we observed the protective effects of baicalein on depressive-like behavior by alleviating neuroinflammation corresponding to the downregulation of the NF- $\kappa B$ pathway.

\section{Methods}

\section{Experimental design}

Experiment I: assessment of behavioral tests, glial alterations, neuroinflammation, and neurochemical changes in the hippocampus of mice induced by EAP Mice were randomly assigned to two groups ( $\mathrm{n}=10$ /group), including the normal control group and EAP group. Mice in each group were tested for tactile allodynia every ten days after the initial immunization. One week after the secondary immunization, mice received behavioral tests and MRI measurements. Then, mice were sacrificed for measurement of biochemical parameters (please see Figure 1A).

\section{Experiment 2: efficacy and molecular mechanism of baicalein on depressive-like behavior and hippocampal neuroinflammation induced by EAP}

As illustrated in Figure 8A, mice were randomly divided into three groups ( $\mathrm{n}=10 /$ group). The first group was the control group, while the remaining two groups subjected to EAP model establishment and treated simultaneously with the same dosage of either saline or baicalein (ie, $100 \mathrm{mg} / \mathrm{kg} /$ day, diluted in $0.1 \%$ dimethyl sulfoxide, DMSO) for one week. Mice in each group received behavior tests and were sacrificed for measurement of biochemical parameters. 
A

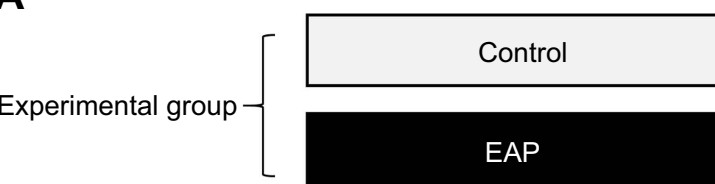

C

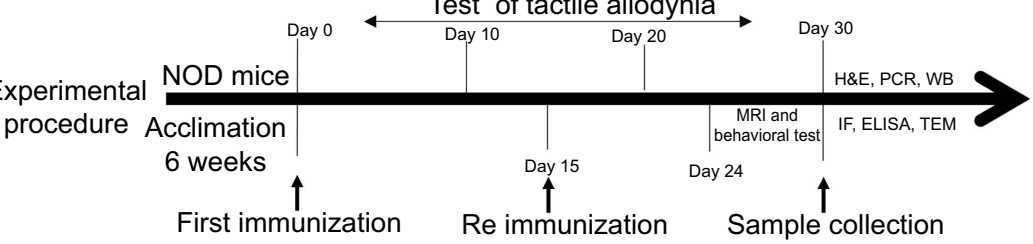

B

Control

EAP

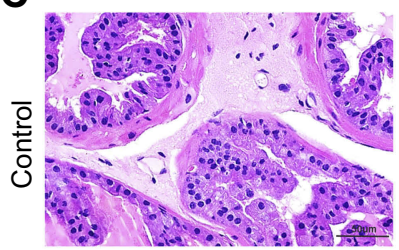

$\underset{\mathrm{U}}{\stackrel{0}{4}}$
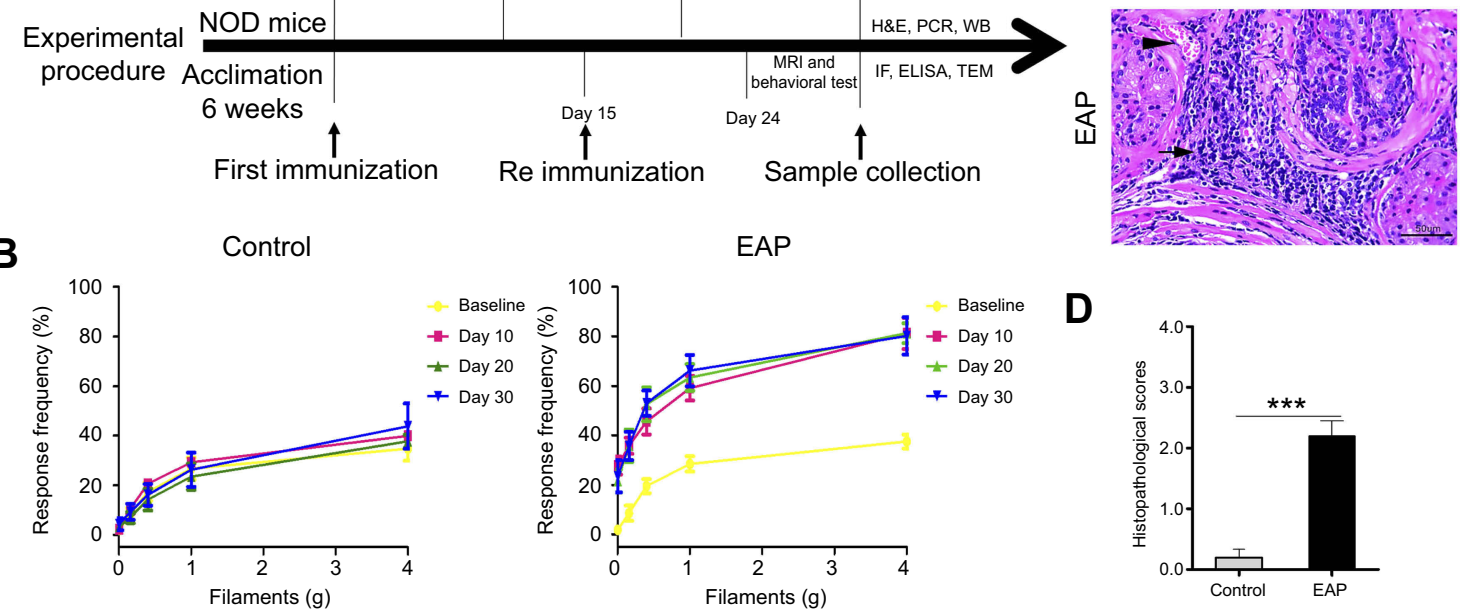

Figure I Establishment of EAP model. (A) The design of experiment I. (B) Chronic pelvic pain was assessed by testing tactile allodynia in the control group and EAP group. (C) Hematoxylin and eosin staining of the prostate. Inflammatory cell infiltration (arrow) and vascular congestion (arrowhead) were observed in the EAP group. Scale bar is $20 \mu \mathrm{m}$. (D) Mean inflammation score of mice in the two groups. ${ }^{*} * p<0.00$ I, vs Control.

Abbreviation: EAP, experimental autoimmune prostatitis.

\section{Animal model}

Non-obese diabetic (NOD) mice are genetically susceptible to EAP and commonly used in the EAP induction. ${ }^{24,25}$ In this study, male NOD/ShiLtJ mice were purchased from the Nanjing Model Animal Center (Nanjing, China). Mice were bred under specific pathogen-free (SPF) conditions at the animal house of Anhui Medical University and used at 6 weeks of age $(16 \sim 19 \mathrm{~g})$. Animal experiments were approved by Institutional Animal Care and Use Committee of Anhui Medical University (No. LLSC201800488). EAP was induced by using standard methodology as previously reported. ${ }^{25}$ In brief, mice were immunized with rat prostate antigen plus complete Freund's adjuvant (CFA; Sigma-Aldrich, Saint Louis, USA) on days 0 and rat prostate antigen plus incomplete Freund's adjuvant (IFA; Sigma-Aldrich, Saint Louis, USA) on days 15. Each mouse was subcutaneously injected in the bilateral foot pad $(0.050 \mathrm{~mL})$, base of the tail $(0.050 \mathrm{~mL})$, and shoulder $(0.050 \mathrm{~mL})$. Chronic pelvic pain was assessed by using Von Frey filaments (North Coast Medical, Encinitas, USA) with forces of 0.04, $0.16,0.4,1$, and 4 grams. The tactile allodynia measurement was performed on the lower abdomen of mice at day 0 (baseline) and every 10 days following the first immunization.

\section{Behavioral analysis}

To investigate the differences in depressive-like (and anxiety-like) behavior in each group, we used four classical methods, including the open field test (OFT), elevated plus maze (EPM), forced swim test (FST), and tail suspension test (TST) in our animal model. Behavioral procedures were conducted in a quiet room under an illumination of 75 lux. Mice were habituated to the experimental environment for two hours prior to the assessment. For each test, the behaviors of mice were evaluated by two raters who were blinded to the experimental design.

The OFT was used to evaluate anxiety-like traits in animals. ${ }^{26}$ The apparatus is composed of a black plastic cage and an overhead digital camera. During testing, mice were initially placed in the same orientation, and the video camera monitored them for $5 \mathrm{~min}$. Exploratory behaviors, including time spent in the center, distance moved, mean speed, and rearing number were analyzed 
by a mouse spontaneous activity video analysis system (Jiliang software technology, Shanghai, China). After each test was finished, the apparatus was cleaned with ethanol.

The EPM test was applied to measure anxiety-like behavior in accord with a previously described method. ${ }^{27}$ The maze positioned $40 \mathrm{~cm}$ above the floor consists of a center platform $(5 \times 5 \mathrm{~cm})$ and four $30 \times 5 \mathrm{~cm}$ arms (two open arms and two closed arms). Mice were individually placed on the EPM, and their behaviors, including the frequency of entries and time spent in each of the four arms, were recorded by video tracking software (Xinxin information, Shanghai, China) for $5 \mathrm{~min}$. After each test was finished, the apparatus was cleaned with ethanol.

The FST was used to analyze depressive-like behavior as previously described. ${ }^{28}$ It was conducted using a clear glass cylinder ( $30 \mathrm{~cm}$ high and $12 \mathrm{~cm}$ diameter) containing $25 \mathrm{~cm}$ of water. Mice were individually allowed to swim for $6 \mathrm{~min}$, and their activities were videotaped. The duration of immobility was defined as the time spent by the mouse floating in the water without struggling and recorded in the last $4 \mathrm{~min}$ of the test. The water was renewal at the end of each test.

The TST method was performed to show alternate periods of immobility when mice were suspended by their tails. ${ }^{29}$ Mice were separately suspended $50 \mathrm{~cm}$ above the floor approximately $1 \mathrm{~cm}$ from the tip of the tail. Each mouse was allowed to hang for $6 \mathrm{~min}$, and the duration of immobility was noted in the last 4 min of the test. Immobility was considered a stationary state without any movement. After each test was finished, the apparatus was cleaned with ethanol.

\section{Histopathology}

Prostate glands were excised and fixed in $10 \%$ paraformaldehyde solution on the 30th day after initial immunization. The necessary steps of histopathology examination include dewaxing, dyeing, dehydration, transparency and sealing. The severity of prostatic inflammation was quantified by using a score of $0-3$ as previously described: ${ }^{24} 0$, no inflammation; 1 , mild perivascular cuffing with slight mononuclear infiltrates; 2, moderate perivascular cuffing with modest mononuclear infiltrates; 3, apparent perivascular cuffing, hemorrhage, and intensive mononuclear cells. Two experienced pathologists independently scored the slides and computed the mean grade of each section.

\section{Immunofluorescence studies}

Brain tissues were fixed for $24 \mathrm{~h}$ and subsequently embedded in paraffin. They were cut into $12-\mu \mathrm{m}$ thick sections, dewaxed and hydrated in decreasing concentrations of ethanol, and subjected to antigen retrieval. Then, $3 \%$ hydrogen peroxide was used for quenching endoperoxide activity, and $5 \%$ bovine serum albumin (BSA, Servicebio, Wuhan, China) was used for blocking nonspecific sites for $1 \mathrm{~h}$. Sections were washed with phosphate buffer and then stained for glial fibrillary acidic protein (GFAP; Elabscience, Wuhan, China) and ionized calciumbinding adapter molecule 1 (Iba1; Abcam, Cambridge, MA, USA) overnight at $4{ }^{\circ} \mathrm{C}$ in the dark at 1:50 dilutions, followed by FITC-conjugated secondary antibodies (Abcam) diluted 1:200. Then, the slides were incubated with DAPI (1:5000; Sigma-Aldrich). Finally, positive cells were observed under a fluorescence microscope (Nikon, Tokyo, Japan). The number of activated microglia and the percentage of GFAP-stained area per image $(\times 400)$ in the hippocampus were analyzed by two blinded observers using the NIH ImageJ software.

\section{Western blot analysis}

Hippocampus tissues were homogenized twice at high speed for $15 \mathrm{~s}$ in slurry tubes with magnetic beads. The extraction buffer containing total protein consisted of RIPA lysis buffer (Thermo Scientific, Waltham, MA, USA) and protease inhibitor cocktail (Beyotime, Shanghai, China). Nuclear protein extraction carried out in accordance with a Nuclear and Cytoplasmic Protein Extraction Kit (Beyotime). Protein concentrations were quantified using the BCA method according to the manufacturer's instructions (Beyotime).

When immunoblotting, equal amounts of protein were subjected to electrophoresis separation and transferred to polyvinylidene fluoride membranes. Subsequently, the membranes were incubated with 5\% milk for $1 \mathrm{~h}$. Then, the membranes were further incubated overnight at $4{ }^{\circ} \mathrm{C}$ with the following antibodies: GFAP (1:800, Elabscience), Iba-1 (1:800, Abcam), NF-к B p65 (1:1000, Elabscience), p-IкB (1:1000, Elabscience), and IкB (1:1000, Elabscience). For total proteins, GAPDH (1:5000, Elabscience) was used as a loading control. For nuclear protein, lamin $\mathrm{A} / \mathrm{C}$ (1:1000, Elabscience) was used as a loading control. Next, the membranes were washed in TBST and incubated with a secondary antibody (anti-rabbit or anti-mouse IgG, 1:5000, Elabscience) for $1 \mathrm{~h}$. Finally, the 
membranes were washed again, followed by signal development using the enhanced chemiluminescence (ECL) system (ChemiScope 5600; Hengmei Technology, Shandong, China). Bands were quantified with NIH ImageJ software and the expression of the protein examined was normalized to the control.

\section{Transmission electron microscopy}

For the transmission electron microscopy (TEM) procedure, the same part of the hippocampus was perfused with $2.5 \%$ glutaraldehyde solution (Solarbio technology, Beijing, China) and fixed with $2.5 \%$ glutaraldehyde. After the fixation and dehydration procedures, hippocampal tissues were embedded in polymeric resins, followed by sectioning and staining with 3\% uranyl acetate and lead citrate. Finally, the slices were observed using TEM (HT-7700; Hitachi, Japan). The features of microglia and astrocyte were identified and distinguished based on a previously reported method. ${ }^{30}$ Glial cells were randomly selected and the number of lysosomal/organelles in the cytoplasm were calculated by two blinded observers.

\section{Real-time PCR}

Total RNA was extracted from the hippocampal tissues with TRIzol reagent (Invitrogen, New York, USA) following the manufacturer's instructions. First-strand cDNA synthesis was performed by using a FastQuant RT Kit (Tiangen Biotech, Beijing, China). Subsequently, quantitative real-time PCR was performed by using SuperReal PreMix Plus Kit (Tiangen Biotech) and an ABI 7500 Real-time PCR system (Applied Biosystems, USA). The primers used were as follows: TNF- $\alpha$ (F 5' CCCTCACA CTCAGATCATCTTCT 3'; R 5' GCTACGACGTGGGCT ACAG 3'), IL-1 $\beta$ (F 5' GAAATGCCACCTTTTGACAG TG 3'; R 5' TGGATGCTCTCATCAGGACAG 3'), IL-6 (F 5' CTGCAAGAGACTTCCATCCAG 3'; R 5' AGTGGT ATAGACAGGTCTGTTGG 3'), IL-8 (F 5' CAAGGCTG GTCCATGCTCC 3'; R 5' TGCTATCACTTCCTTTCTGT TGC 3'), IL-18 (F 5' GACTCTTGCGTCAACTTCAAGG 3'; R 5' CAGGCTGTCTTTTGTCAACGA 3'), SLC6A4 (F 5' CTCCGCAGTTCCCAGTACAAG 3'; R 5' CACGG CATAGCCAATGACAGA $3^{\prime}$ ), IDO (F 5' TGGCGTATG TGTGGAACCG 3'; R 5' CTCGCAGTAGGGAACAGC AA 3'), and GAPDH (F 5'-AGGTCGGTGTGAACGGA TTTG 3'; R 5' GGGGTCGTTGATGGCAACA 3'). All reactions were conducted in a $20 \mu \mathrm{L}$ tube, and mouse GAPDH was used as an internal standard. The formula $2^{-\Delta \Delta} \mathrm{Ct}$ method was used for each sample in data analysis.

\section{ELISA}

Serum serotonin concentrations were determined by using a Serotonin/5-Hydroxytryptamine ELISA Kit (Elabscience) according to the manufacturer's protocol. Multiwall plates were coated with $50 \mu \mathrm{L}$ per well of serum samples or standard solution. Next, $50 \mu \mathrm{L}$ per well of biotinylated detection antibody solution was immediately added to multiwall plates, incubated at $37^{\circ} \mathrm{C}$ for $45 \mathrm{~min}$, and washed with washing buffer. Then, $100 \mu \mathrm{L}$ horseradish peroxidase (HRP) conjugate solution was added, incubated at $37^{\circ} \mathrm{C}$ for $30 \mathrm{~min}$, and washed again. Later, $90 \mu \mathrm{L}$ substrate reagent (TMB) was added, incubated at $37{ }^{\circ} \mathrm{C}$ for $15 \mathrm{~min}$, and washed. Finally, $50 \mu \mathrm{L}$ stop solution was added. The optical density values of each well were measured at $450 \mathrm{~nm}$ using a microplate reader, and the mean concentrations of each sample were calculated.

\section{In vivo high magnetic field $M R I$ and proton MRS}

T2-weighted and ${ }^{1} \mathrm{H}$-MRS images were acquired in a Bruker 9.4 T MRI scanner (Bruker BioSpin MRI, Ettlingen, Germany). Mice were anesthetized with isoflurane and placed in a supine position on an MRI-compatible cradle, with controlled respiration at 30 breaths per min. The use of a 3-slice scout scan by scanning a fast lowangle shot sequence ensured that each mouse was placed in the proper position in relation to the magnet and coil isocenter. T2-weighted images were obtained by using a two-dimensional rapid acquisition with relaxation enhancement sequence in the coronal plane (parameters: repetition time $\mathrm{TR}=9500 \mathrm{~ms}, \mathrm{TE}=36.8 \mathrm{~ms}$, and slice thickness $=0.5 \mathrm{~mm}$ ).

For ${ }^{1} \mathrm{H}-\mathrm{MRS}$, the volume of interest (VOI) was placed in the same location as the left hippocampus $\left(1 \times 1 \times 1.5 \mathrm{~mm}^{3}\right)$. The first- and second-order shims over the VOI were adjusted by a fast shimming procedure (FASTMAP). High-quality, single voxel ${ }^{1} \mathrm{H}-\mathrm{MRS}$ was performed for $45 \mathrm{~min}$ by using a point-resolved spectroscopy (PRESS) sequence. The parameters are as follows: $\mathrm{TR}=2500 \mathrm{~ms}, \mathrm{TE}=2.8 \mathrm{~ms}, 1000$ accumulations, and excitation pulse bandwidth $=4 \mathrm{kHz}$. In addition, field maps were obtained for shimming and variable pulse power, and an optimized relaxation delay (VAPOR) protocol was used for water suppression. The unsuppressed water spectrum was also obtained for reference. During postprocessing, MRS data were analyzed using an advanced AMARES 
algorithm supported by the J-Magnetic Resonance User Interface (JMRUI, version 6.0 beta) software package.

\section{Baicalein treatment}

Baicalein (Sigma-Aldrich) was used as an anti-inflammatory agent to confirm whether EAP-induced depressive-like behavior was improved and its underlying mechanism if it did. Baicalein treatment was started at the last week of the EAP model, and baicalein was dissolved in 0.1\% DMSO (Saiguo biotech, Guangzhou, China) and administered by oral gavage once per day in a dosage of $100 \mathrm{mg} / \mathrm{kg}$. In other groups, mice were administered an equal volume of $0.9 \%$ saline in DMSO.

\section{Statistical analysis}

Statistical analysis was performed by using SPSS version 16.0 (IBM-SPSS Inc. Chicago, IL, USA). The results are expressed as the mean \pm standard error of the mean. Comparisons between any two groups were performed using independent sample $t$-test. Linear dependencies between measured behavioral parameters and serotonin concentrations were identified by Pearson correlation coefficients (r). For numeric variables among three groups, one-way analysis of variance (ANOVA) was used to compare the parametric variables. A value of $p<0.05$ was considered significant.

\section{Results}

\section{EAP induces depression-like behavior in mice}

To clarify that depressive-like behavior was induced by EAP, we first established and evaluated the mouse model of EAP. As shown in Figure 1B, an increase in frequency in responses to an abnormal pain stimulus was exhibited by mice in the EAP group. This result revealed that mice developed pelvic pain symptoms following the EAP procedure. Moreover, histological examination of the prostate in the model group showed stromal lymphocytic infiltrations, vascular congestion, and gland destruction (Figure 1C). In accordance with these findings, Figure 1D displayed a higher pathological score for prostates in the EAP group $(p<0.001)$. A combination of these results indicated successful establishment of the EAP model.

Then, the behavioral performances of mice were assessed by the OFT, EPM FST, and TST. In the OFT, EAP mice exhibited obvious anxiety-like behavior as shown by the decreased time in the center region, distance in the center region, and number of rearings $(p<0.05$ or $p<0.001$; Figure 2A and B). Consistent with these results, EAP mice showed a marked decrement in the open arm time, percentages of open arm time, and open arm entries $(p<0.05$ or $p<0.01$; Figure $2 \mathrm{C}$ and $\mathrm{D})$. When mice were exposed to the FST, a significant increment in the duration of immobility was found in the EAP group $(p<0.05$; Figure 2E). Additionally, the induction of depression-like behavior was also shown in the TST, with EAP mice spending more immobility time $(p<0.05$; Figure $2 \mathrm{~F})$. As noted above, these results demonstrated the induction of depression-like (and anxiety-like) behavior following EAP.

\section{EAP causes microglial activation and reactive astrocytosis in the hippocampus}

To investigate the changes in the morphology and functions of microglia and astrocyte induced by EAP, we applied multimodal detections of hippocampal glial cells. As illustrated in Figure 3A, coexpression of GFAP (for astrocytes) and Iba1 (for microglia) was detected in the CA1, CA3, and DG regions in the hippocampus using immunofluorescence. Both the number of activated microglial cells and the percentages of GFAP-stained areas for astrocytes were significantly larger in all three hippocampal regions in the EAP group ( $p<0.05$ or $p<0.01$ or $p<0.001$; Figure $3 \mathrm{~B}$ ). We also analyzed the protein levels of Iba-1 and GFAP by Western blotting (Figure 3C). Consistent with immunofluorescence analysis, the protein levels of both Iba-1 and GFAP significantly increased in the hippocampus of EAP mice $(p<0.05$ or $p<0.01$ ).

In addition, we observed the ultrastructural features of microglia and astrocytes in the hippocampus of mice using TEM. Compared with the control group, the EAP group showed more microglial activation as evidenced by increased and enlarged lysosomes in the cytoplasm $(p<0.01$; Figure $4 \mathrm{~A}-\mathrm{C})$. Similar to microglia in the EAP model, astrocytes also showed intense activation characterized by increased cytosol in the cytoplasm $(p<0.001$; Figure 4D-F). Briefly, the changes in structure and function in glial cells confirmed that EAP caused microglial activation as well as reactive astrocytosis in the hippocampus.

\section{EAP increases the expression of proinflammatory markers in the hippocampus}

To understand the involvement of microglial activation in neuroinflammation, we measured the mRNA expression 
A

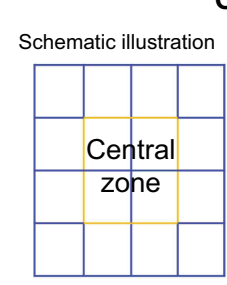

B
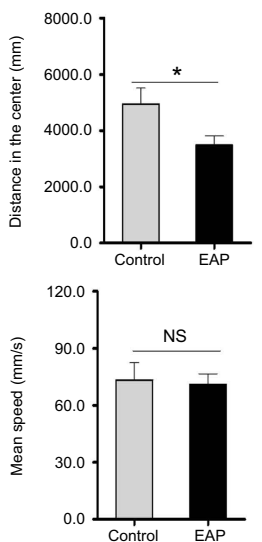

Open field test

Control
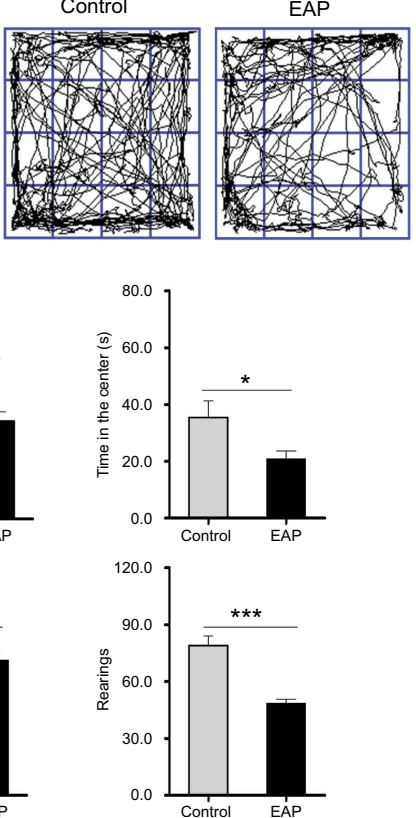

C

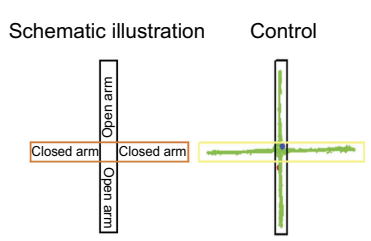

D
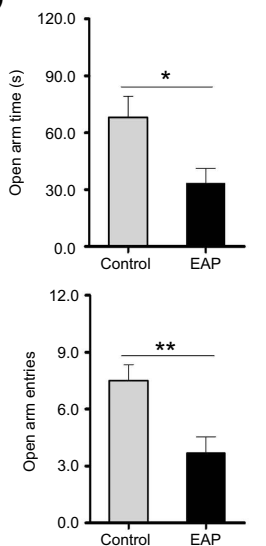

E

Forced

swimming

test

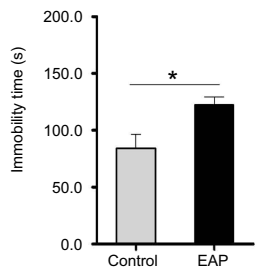

F

Tail

suspension

test
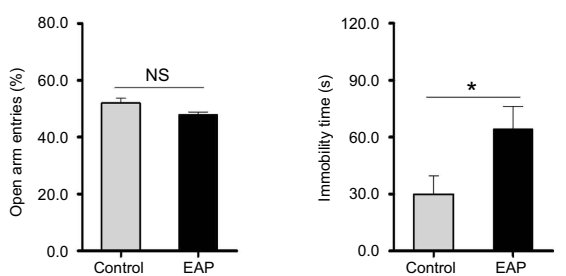

Figure 2 EAP induces depression-like behavior in mice. (A) Schematic illustration and representative track plots of mice during the OFT. (B) Distance in the center, time in the center, mean speed, and rearing number in the OFT. (C) Schematic illustration and representative track plots of mice during the EPM test. (D) Open arm time, percentages of open arm time, open arm entries, and percentages of open arm entries in the EPM test. (E) Time spent immobile in the FST. (F) Time spent immobile in the TST. $* p<0.05, * * p<0.01$, and $* * * p<0.001$, vs Control.

Abbreviations: EAP, experimental autoimmune prostatitis; OFT, open field test; EPM, elevated plus maze; FST, forced swim test; TST, tail suspension test; NS, nonsignificant.

A

Control
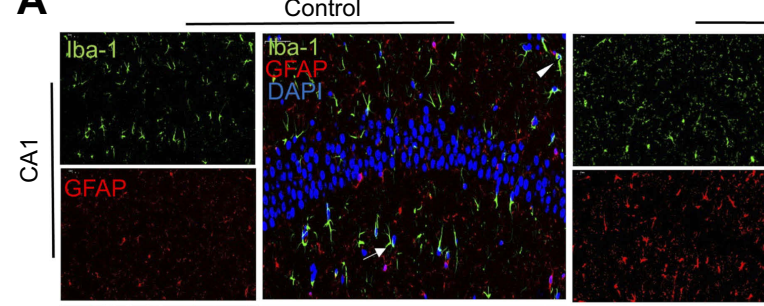

EAP
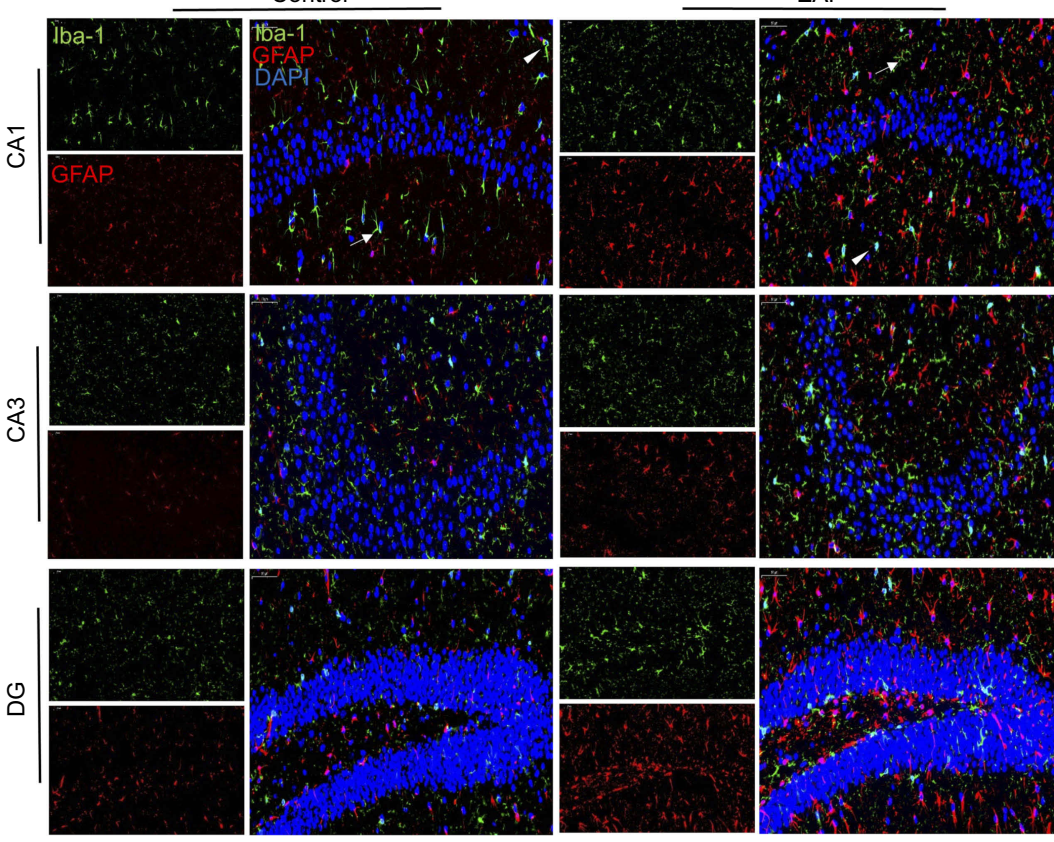
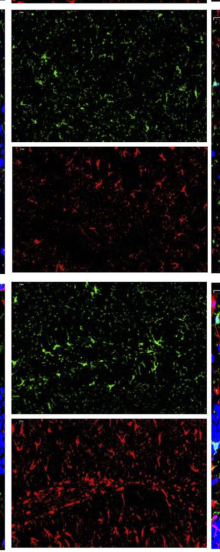
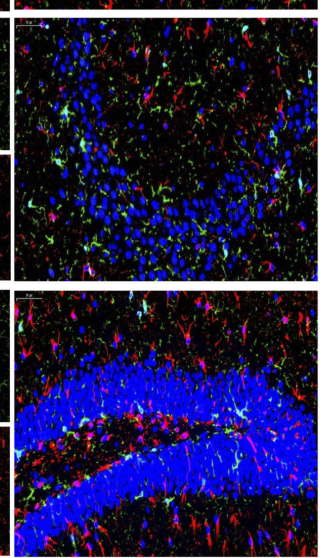

B
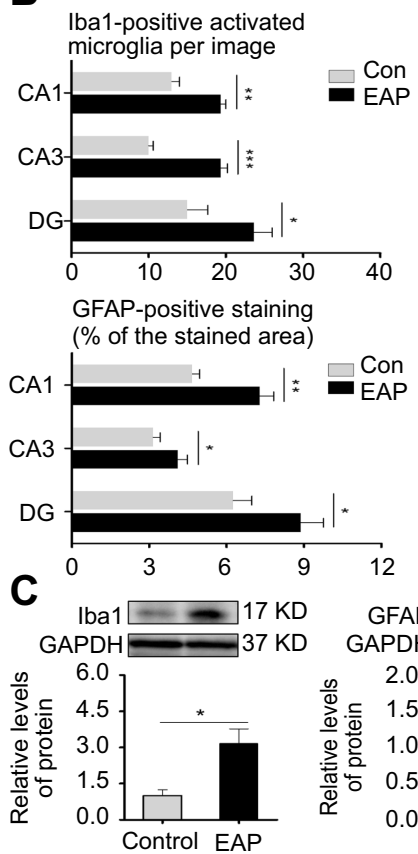

$$
\square \text { Con }
$$

EAP

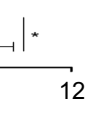

GFAP $=50 \mathrm{KD}$ GAPDH $237 \mathrm{KD}$

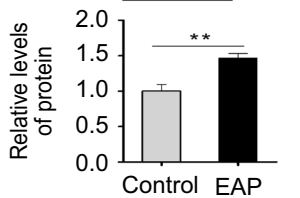

Figure 3 EAP increases the expression of microglial and astrocytic markers in the hippocampus. (A) Immunofluorescence staining of CAI, CA3, and DG regions in the hippocampus. Ibal (green), GFAP (red), DAPI (blue); scale bar is $50 \mu \mathrm{m}$. White arrow and arrowheads show resting microglia and activated microglia respectively. (B) Qualification of Iba-I-positive cells and the percentages of GFAP-stained areas in the CAI, CA3, and DG regions in the hippocampus. (C) Western blot analysis of Iba-I and GFAP. ${ }^{*} p<0.05$, $* * p<0.01$, and $* * * p<0.001$, vs Control.

Abbreviations: EAP, experimental autoimmune prostatitis; Ibal, ionized calcium-binding adapter molecule I; GFAP, glial fibrillary acidic protein. 


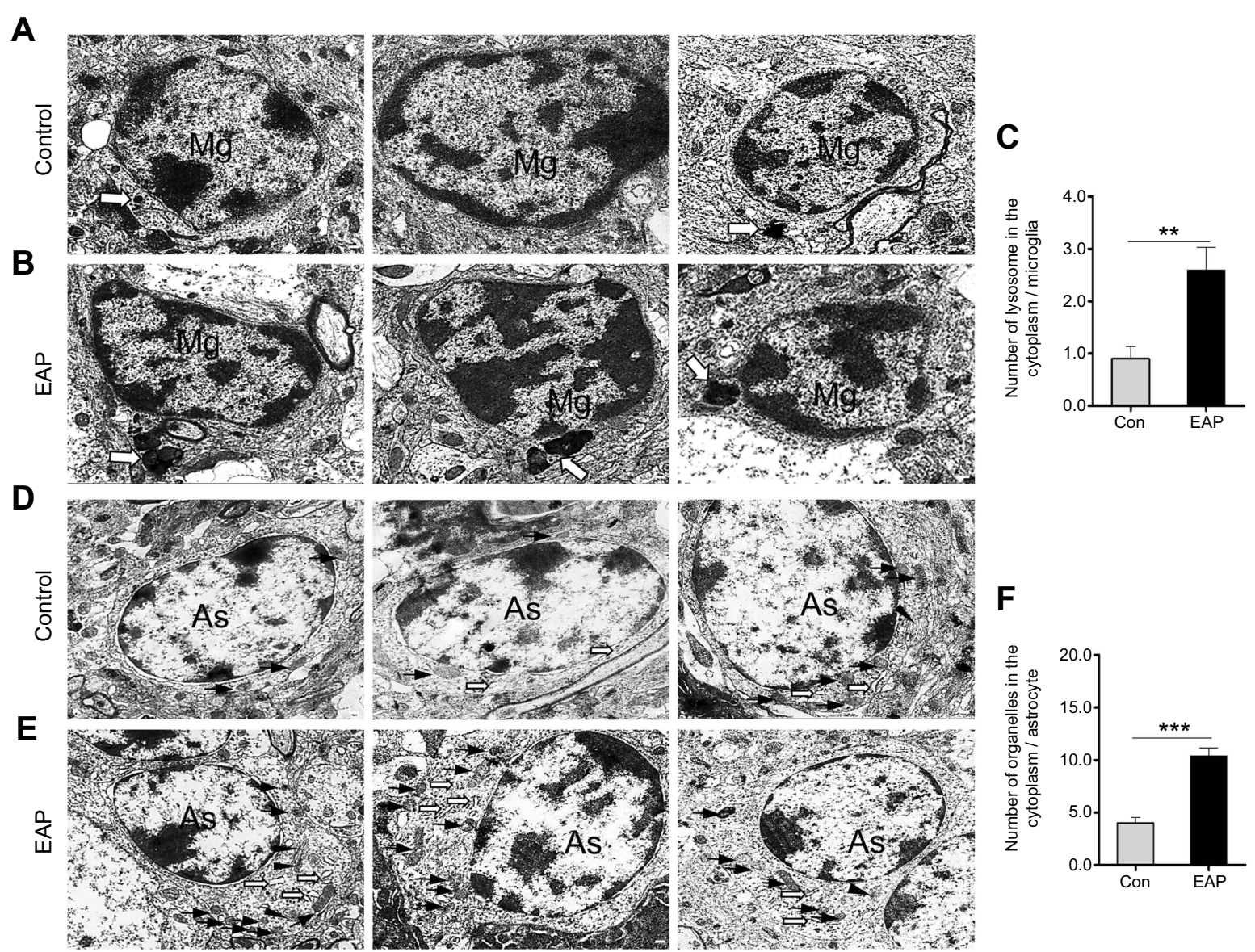

Figure 4 Ultrastructural changes in hippocampal glial cells. (A) TEM observation shows a normal appearance of microglial cells with a primary lysosome (hollow arrow) in the control group. (B) Microglia activation in the EAP group was evidenced by increased and enlarged lysosomes (hollow arrow) in the cytoplasm. (C) Quantitative analysis of the number of lysosomes in the cytoplasm of microglia. (D) The control group showed normal astrocyte morphology with a few mitochondria (arrows) in the cytoplasm. (E) Reactive astrocytosis characterized by increased cytoplasmic Golgi body (arrowheads), endoplasmic reticulum (hollow arrows), and mitochondria (arrows) in the EAP group. (F) Quantitative analysis of the number of organelles in the cytoplasm of microglia. Scale bar is $200 \mathrm{~nm}$. $* * p<0.01$, and $* * * p<0.00 \mathrm{I}$, vs Control.

Abbreviations: TEM, transmission electron microscopy; EAP, experimental autoimmune prostatitis; Mg, microglia; As, astroglia.

levels of multiple proinflammatory mediators in the hippocampus, including TNF- $\alpha$, IL-1 $\beta$, IL-6, IL-8, and IL18, by RT-PCR. As shown in Figure 5A-E, except for IL-18 ( $p=0.15)$, significantly higher gene expression levels of these cytokines were detected in EAP-induced animals than in control animals $(p<0.05$ or $p<0.01)$. These results indicated that EAP induced neuroinflammation in the hippocampus.

\section{EAP influences serotonin transport and biosynthesis in the hippocampus}

Depression is reportedly related to decreased serotonin in the CNS. The enzyme SLC6A4 and IDO, which play a key role in serotonin transport and biosynthesis, are sensitive to proinflammatory cytokines. ${ }^{31,32}$ Therefore, to further study the impact of EAP-induced neuroinflammation on serotonin metabolism, we measured the gene expressions of SLC6A4 and IDO by RT-PCR. As expected, EAP mice exhibited higher expression levels of both SLC6A4 and IDO in the hippocampus $(p<0.05$; Figure $6 \mathrm{~A})$. These observations were also accompanied by a decreased serum serotonin level in the EAP group $(p<0.05$; Figure 6B). In addition, as shown in Figure 6C, the serotonin concentrations in EAP mice were positively correlated with time in the center in the OFT $(\mathrm{r}=+0.692$; $p=0.027)$ and open arm time in the EPM $(\mathrm{r}=+0.657$; $p=0.039)$ and negatively correlated with immobility time in the FST $(\mathrm{r}=-0.733 ; p=0.016)$ and TST $(\mathrm{r}=-0.755$; $p=0.012$ ). These data suggest that the reduction in serotonin transport and biosynthesis lowered the serotonin concentration, which could have a critical role in the formation of EAP-induced depression. 

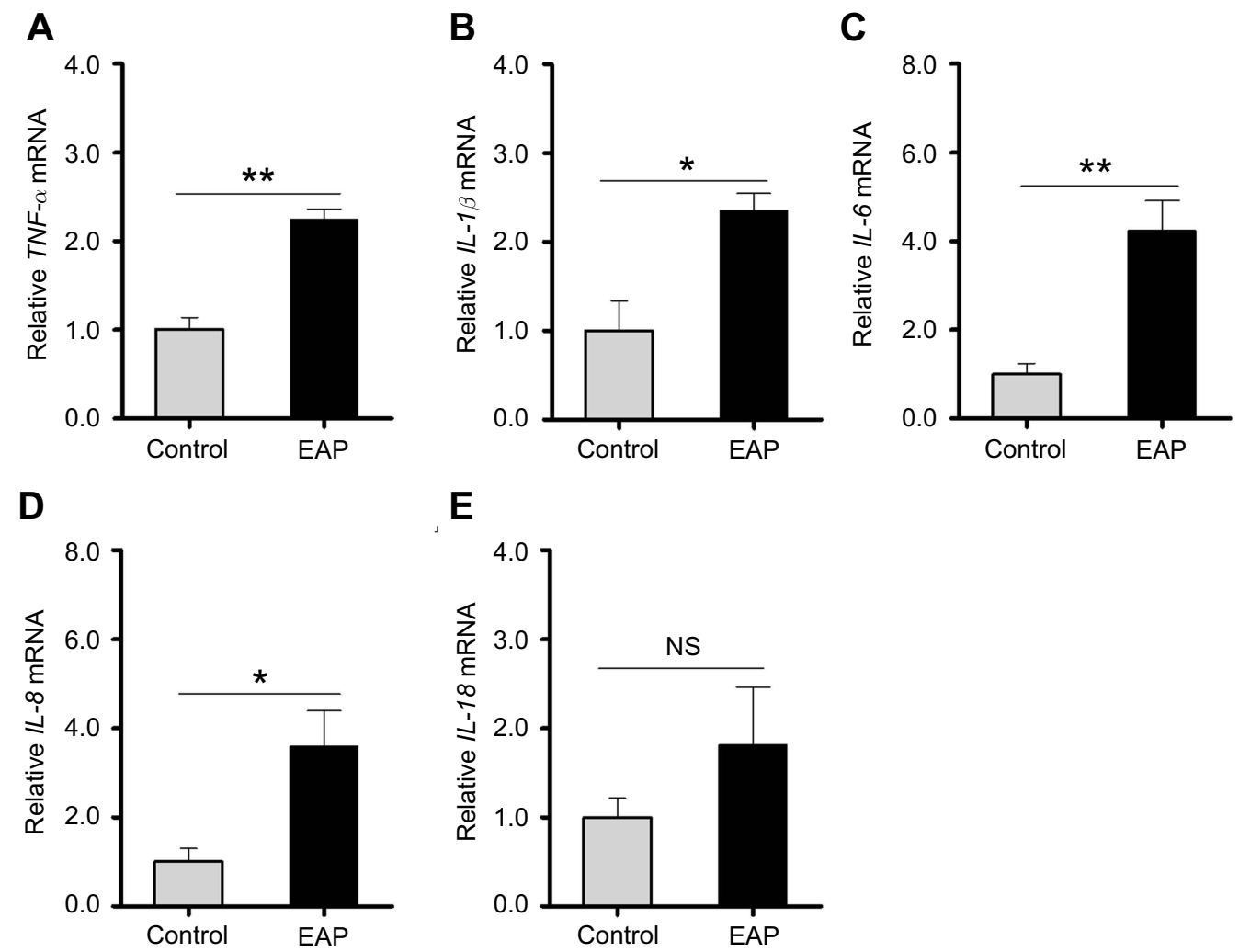

Figure 5 Detection of cytokine changes in the hippocampus of control and EAP mice. (A-E) Real-time PCR analysis of TNF- $\alpha$, IL-I $\boldsymbol{\beta}$, IL-6, IL-8, and IL-I8. * $p<0.05$, $* * p<0.01$, vs Control.

Abbreviations: EAP, experimental autoimmune prostatitis; PCR, polymerase chain reaction; TNF- $\alpha$, tumor necrosis factor- $\alpha$; IL-I $\beta$, interleukin- I $\beta$; IL-6, interleukin-6; IL-8 interleukin-8; IL-18, interleukin-18; NS, nonsignificant.

\section{EAP alters glutamate levels in the hippocampus}

As we observed microglial activation and subsequent cytokine releasing and changes in the serotoninergic system in our animal model, high-field MRI scanning further provided a useful noninvasive approach to identify hippocampal glutamate alterations that were related to the pathology of depression and mediated by proinflammatory factors. For anatomic structure, representative T2-weighted images for both the EAP group and control group are displayed in Figure 7A, but there was no obvious difference in the hippocampus. Nonetheless, ${ }^{1} \mathrm{H}-\mathrm{MRS}$ spectra acquired from the corresponding area within the left hippocampus exhibited differences in specific neurochemicals between the two groups (Figure 7B). In contrast to the control group, the EAP group showed a significantly increased taurine ( Tau)/total creatine ( $\mathrm{tCr}$, creatine + phosphocreatine) ratio, while the glutamate + glutamine $(\mathrm{Glx}) / \mathrm{tCr}$ ratio was decreased $(p<0.05$; Figure $7 \mathrm{C})$. These results indicate that abnormalities in the glutamatergic system may be involved in EAP-induced depressive-like behavior.

\section{Baicalein treatment alleviates EAP- induced depressive-like behavior and neuroinflammation through the NF- $\kappa B$ pathway}

Baicalein served as a common inhibitor of inflammation and was used to assess the therapeutic effect in treating EAP-induced depressive-like behavior in this study. As a result, compared to EAP, baicalein treatment markedly increased the time in the center in the OFT $(p<0.05$; Figure 8B). In the EPM, the open arm time in the baicalein treatment group was longer but not significantly different from that in the EAP group ( $p=0.07$; Figure $8 \mathrm{C})$. Moreover, baicalein could significantly normalize the depressive-like behaviors of EAP, which was indicated by less immobility time in the FST and TST $(p<0.05$ or $p<0.01$; Figure 8D and E).

The effect of baicalein on neuroinflammation was checked by examining the mRNA levels of TNF- $\alpha$, IL$1 \beta$, IL-6, and IL-8. As depicted in Figure 8F, baicalein treatment significantly inhibited EAP-induced expressions of those cytokines in the hippocampus $(p<0.05$ or $p<0.01)$. 
A

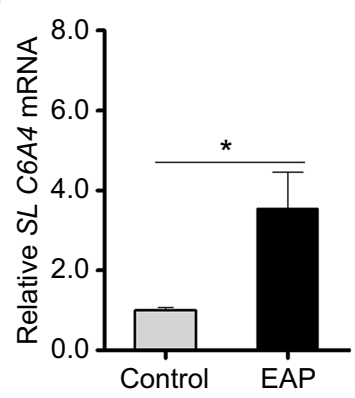

B

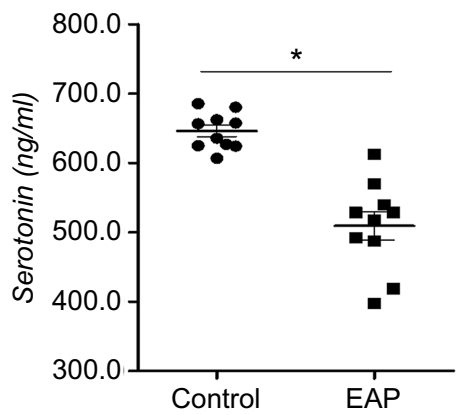

C
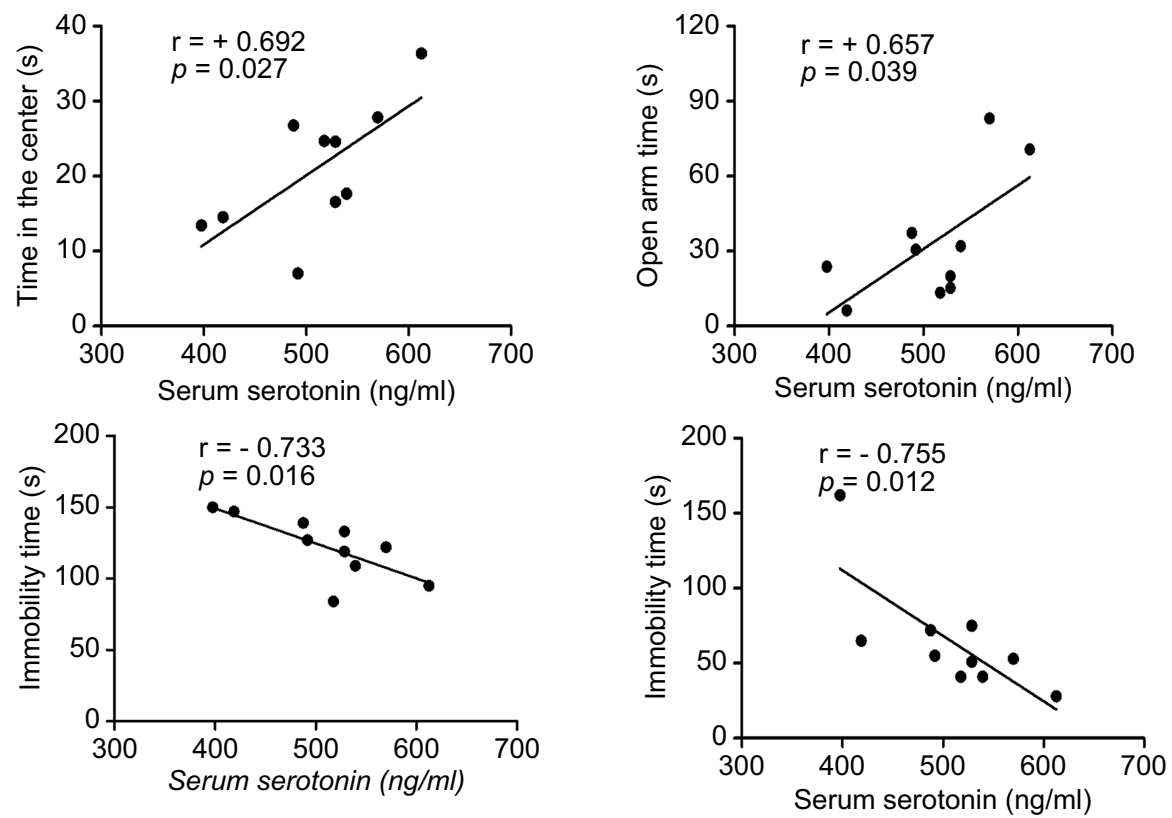

Figure 6 EAP causes increased concentrations of serotonin in the hippocampus of EAP mice. (A) Real-time PCR analysis of SLC6A4 and IDO. (B) ELISA analysis of serum serotonin. (C) Correlations between serum serotonin concentrations and behavioral measurements. r, Pearson correlation coefficient. ${ }^{*} p<0.05$, vs Control.

Abbreviations: EAP, experimental autoimmune prostatitis; PCR, polymerase chain reaction; SLC6A4, serotonin transporter; IDO, indoleamine 2,3-dioxygenase; ELISA, enzyme-linked immunosorbent assay.

Furthermore, the abovementioned anti-inflammatory effect of baicalein may be involved in the reduction in NF- $\kappa B$ pathway activity. Figure $8 \mathrm{G}$ shows NF- $\mathrm{KB}$ P65 activation and degradation of the inhibitory subunit (IאB) in the EAP group. However, this response was dramatically suppressed after baicalein treatment $(p<0.05$ or $p<0.01)$. Taken together, these results suggest that the anti-neuroinflammatory effect of baicalein is related to its actions on downregulation of the NF- $\mathrm{KB}$ pathway.

\section{Discussion}

$\mathrm{CP} / \mathrm{CPPS}$ is potentially an autoimmune disease that causes a variety of inflammatory factors, including IL- $1 \beta$, TNF-a, and IL-6, which can all ultimately damage brain function. Among a significant number of male patients with $\mathrm{CP} / \mathrm{CPPS}$, many of them were substantially bothered by psychological deficits. Particularly, a higher frequency of depressive symptoms was observed in patients with CP/CPPS than in the normal population. Similarly, in animal models, EAP mice become more prone to depressive behavioral changes. In the present study, we established an EAP model on mice and evaluated their depressive-like behaviors by the OFT, EPM, FST, and TST. The results of significant depression-like activity levels observed in the EAP group confirmed a possible link between depressive behavior and the EAP model.

One of the potential pathogenic processes for peripheral immune inflammation-induced depression is that pro-inflammatory cytokines enter the brain and trigger neuroinflammation by activating microglia. ${ }^{33}$ Additionally, neuropathic pain may be associated with depressive behavior. ${ }^{34,35}$ However, with regard to $\mathrm{CP} / \mathrm{CPPS}$-induced depression, whether 
A

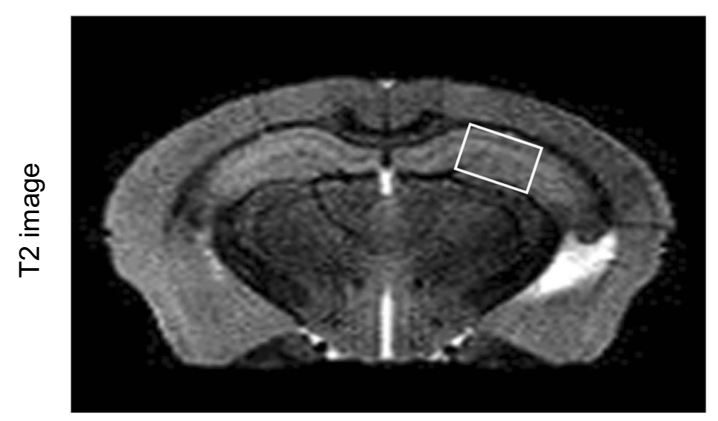

B

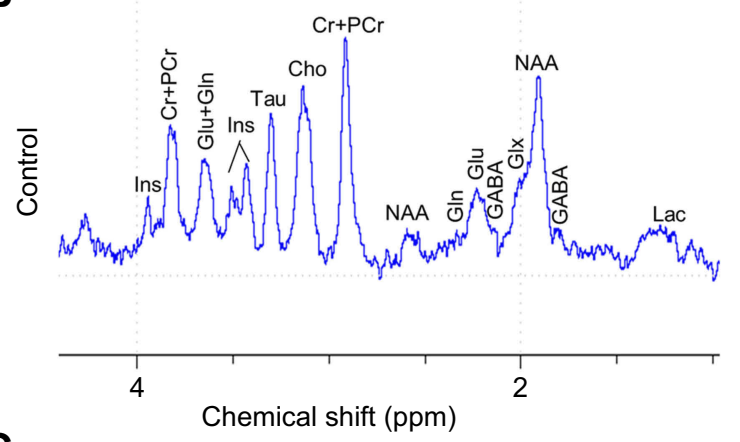

EAP
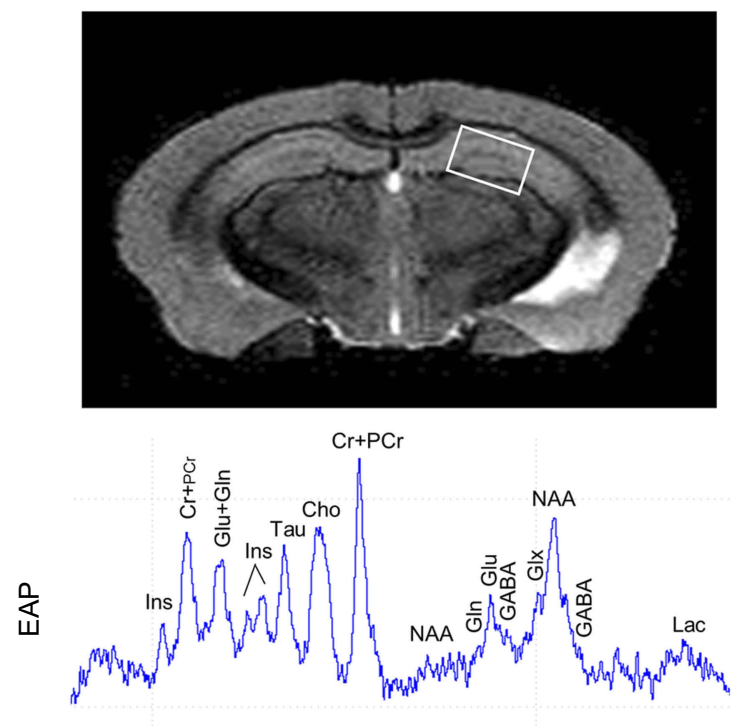

$4 \quad$ Chemical shift (ppm)

C
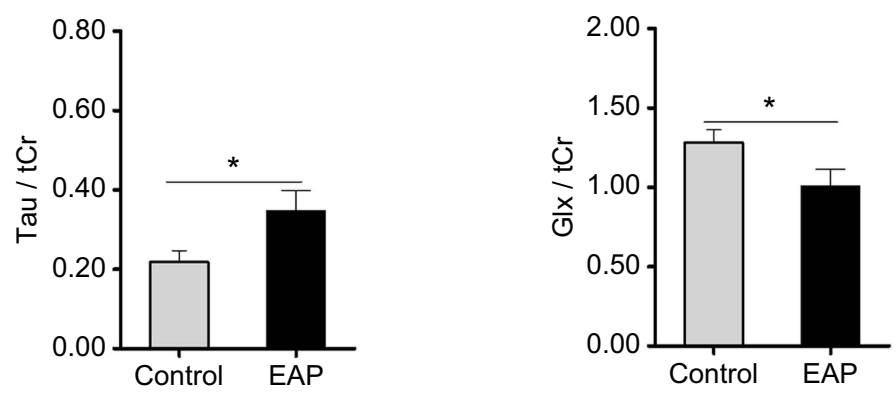

Figure 7 Neurochemical changes in the hippocampus of EAP mice. (A) MRI anatomic images of the brain in mice. The white boxes indicate the placement of the volume of interest. (B) Representative in vivo 'H-MRS. (C) Comparisons of metabolite ratios ( $\mathrm{Gl} / \mathrm{tCr}$ and $\mathrm{Tau} / \mathrm{tCr}$ ) between control and EAP mice in the hippocampus. * $p<0.05$, vs Control.

Abbreviations: EAP, experimental autoimmune prostatitis; MRI, magnetic resonance imaging; 'H-MRS, proton magnetic resonance spectroscopy; Cr, creatine; PCr, phosphocreatine; Glu, glutamate; Gln, glutamine; Tau, taurine; Glx, Glu + Gln; tCr (total creatine, $\mathrm{Cr}+\mathrm{PCr}$ ).

inflammation or pain is the main factor is difficult to determine because $\mathrm{CP}$ is accompanied by CPPS, which manifests as a syndrome that cannot be separated. Thus, we speculated that the combined action of prostate inflammation and neuropathic pain in the pelvis ultimately leads to depression. In the CNS, microglia and astrocyte are the two major sources of innate immunity. Microglial cell is considered as the main regulator in immune responses, while astrocyte plays a neurosupportive role and is essential for regulating neurotransmission. ${ }^{36}$ Activated microglia and astrocyte could mediate effects on depressive-like behavior in rodents. ${ }^{37,38}$ In our model, we discovered that the glial markers Ibal and GFAP were both significantly overexpressed in the EAP group, which implied microglial activation and reactive astrocytosis in the hippocampus. This result was also confirmed by the observation of ultrastructural changes in glial cells. Hence, we deduced that microglial activation, accompanied by reactive astrocytosis, is likely a defining feature of EAP-induced depression.

Neuroinflammation refers to the cellular and biochemical responses to different stimuli that occur in the CNS. ${ }^{6}$ Glial activation is an important component of the entire series of events, which can subsequently mediate the secretion of various proinflammatory cytokines. These overexpressed cytokines interact with changes in neurotransmitter metabolism, neuroendocrine function, and synaptic plasticity. ${ }^{39}$ For this reason, investigators suggest that neuroinflammation acts as an important factor that 
A
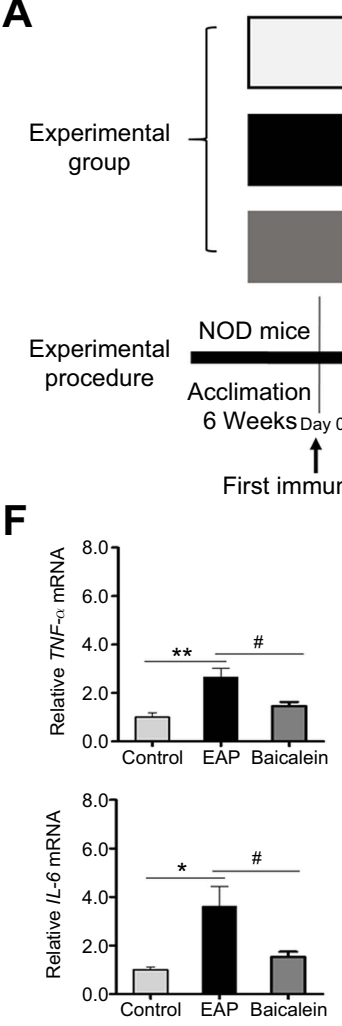

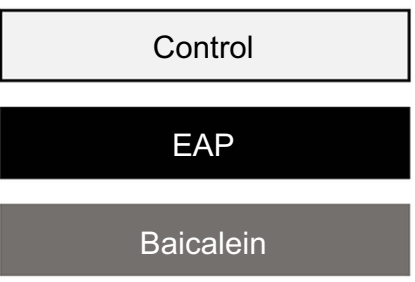

Baicalein
B

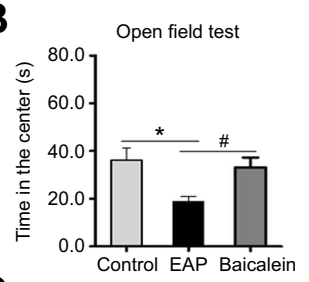

D

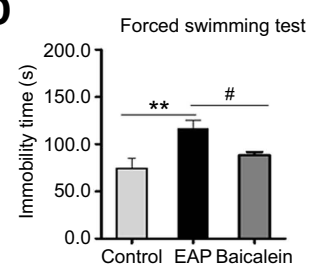

C

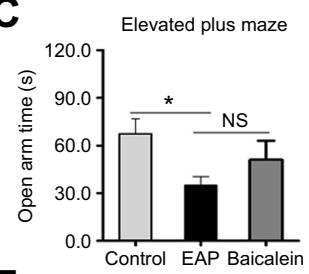

E

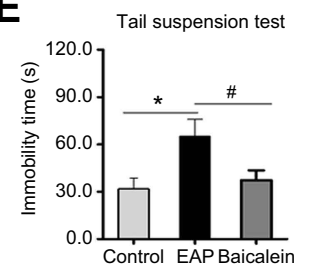

G
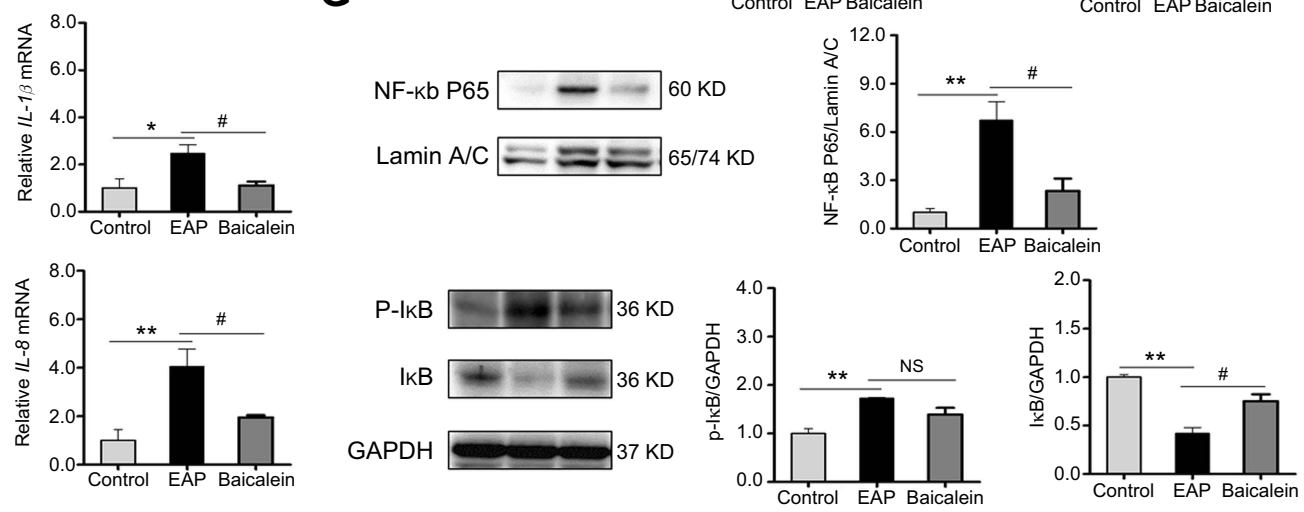

Figure 8 Baicalein treatment reverses EAP-induced depressive-like behavior and neuroinflammation via the NF-kB pathway. (A) The design of experiment 2. (B) Time in the center of the OFT. (C) Open arm time in the EPM. (D) Immobility time in the FST. (E) Immobility time in the TST. (F) PCR analysis of TNF- $\alpha$, IL-I $\boldsymbol{\beta}$, IL-6, and IL-8. (G) Western blot analysis of NF-кB p65, p-ІкB, and ІкB. ${ }^{*} p<0.05$, ${ }^{* *} p<0.01$, vs Control. ${ }^{\#} p<0.05$, vs EAP.

Abbreviations: EAP, experimental autoimmune prostatitis; NF- $\mathrm{kB}$, nuclear factor-kappa B; OFT, open field test; EPM, elevated plus maze; FST, forced swim test; TST, tail suspension test; TNF- $\alpha$, tumor necrosis factor- $\alpha$; IL-I $\beta$, interleukin-I $\beta$; IL-6, interleukin-6; IL-8 interleukin-8; NS, nonsignificant.

contributes to the pathophysiology of depression. In our study, we found significantly increased expression of cytokines, including TNF $\alpha$, IL-1 $\beta$, IL-6, and IL-8, in the EAP group. This result gave us a clue to alleviating the depression process by suppressing neuroinflammation.

Since immune activation plays a crucial role in mediating serotonin and glutamate systems in pathogenesis of depression, we then extended our work to study changes of the neurotransmitters. The serotonin level in the CNS mainly depends on serotonin transport and biosynthesis. SLC6A4 is the most studied gene associated with serotonin transport. This gene regulates the serotonergic system by encoding a membrane integral protein that is involved in the reuptake of serotonin from synaptic clefts to the presynaptic terminal. ${ }^{40}$ The other extensively investigated enzyme that affects serotonin concentration is IDO. As tryptophan is the synthetic material of serotonin and IDO degrades it into kynurenine, IDO serves as the rate-limiting enzyme of serotonin biosynthesis. In our research, as expected, the mRNA expression levels of SLC6A4 and
IDO were both markedly increased in the hippocampus of EAP mice. Subsequently, we further investigated the serum serotonin level and its correlations with behavioral tests in mice. These findings suggested that the serotonin level decreased in EAP mice and significantly correlated with their depressive behavior.

To the best of our knowledge, the current description of the EAP-induced depressive-like mouse model is the first reported instance where neurochemical abnormalities were assessed by high-field MRI. Assessment of the changes in the metabolism of neurochemicals through this technique could help to fully understand the in vivo disease process of depression. Notably, our data indicated a decrease in the hippocampal $\mathrm{Glx} / \mathrm{tCr}$ ratio in the EAP group. It is generally accepted that the glutamate concentration in CNS is closely associated with glutamate release and uptake. The astrocyte plays a key role in these events by regulating glutamate transporters and release of glutamate vesicles. ${ }^{41}$ Meanwhile, microglia has been shown to secret proinflammatory cytokines to modulate the functional status 
of astrocyte. ${ }^{13}$ In our EAP model, glial activation might alter the reuptake and release of glutamate in the hippocampus and lead to decreased glutamate levels, which was the potential reason that contributed to depression.

To improve depressive-like behavior and neuroinflammation induced by EAP, we used baicalein, which has anti-inflammatory and neuro-protective effects. As expected, baicalein administration alleviated EAPinduced depressive-like behaviors in mice and decreased the expressions of several proinflammatory factors in the hippocampus. We reasoned that the antidepressant action of baicalein was mediated by an anti-neuroinflammatory effect. Moreover, NF-кB P65 activation and $\mathrm{I} \kappa \mathrm{B}$ degradation were observed in the baicalein treatment group. Thus, we concluded that baicalein attenuated EAP-induced depressive-like behavior by inhibiting neuroinflammation via suppressing the NF- $\kappa$ B pathway. Evidence of this efficiency provides a novel treatment for ameliorating neuroinflammation-related depression.

There are some insufficiencies in this study. Firstly, although hippocampus is a key structure highly associated with emotional regulation, other brain areas related to mood control, such as frontal cortex and basolateral amygdala, remain to be further investigated. Secondly, this study preliminary illustrates the treatment effect of baicalein on EAP-induced depressive-like behavior in mice. However, whether it exerts the antidepressant effect through attenuating prostatic inflammation or glial activation or both is not clear and needs further study in the future.

\section{Conclusion}

The present study is the first to demonstrate that EAPinduced depressive-like behavior are related to microglia activation with subsequent neurobiological alterations. In addition, baicalein may be a new therapeutic agent in the treatment of $\mathrm{CP} / \mathrm{CPPS}-$ related depression.

\section{Ethics approval and consent to participate}

This study was conducted after obtaining approval of the Institutional Animal Care and Use Committee of Anhui Medical University (No. LLSC201800488), and conformed to the National Institutes of Health Guide for Care and Use of Laboratory Animals.

\section{Acknowledgment}

This work was supported by the Key Project of National Natural Science Foundation of China (81630019) and the National Natural Science Fund of China (81870519, 81470986). We offer special acknowledgement to technician Yun Wu, Lulu Wang, and PhD student Chen Lu for their selfless technical support, encouragement, and guidance during this work.

\section{Author contributions}

All authors contributed to data analysis, drafting and revising the article, gave final approval of the version to be published, and agree to be accountable for all aspects of the work.

\section{Disclosure}

The authors report no conflicts of interest in this work.

\section{References}

1. Krieger JN, Lee SW, Jeon J, et al. Epidemiology of prostatitis. Int $J$ Antimicrob Agents. 2008;31 Suppl 1:S85-S90. doi:10.1016/j. ijantimicag.2007.08.028

2. Habermacher GM, Chason JT, Schaeffer AJ. Prostatitis/chronic pelvic pain syndrome. Annu Rev Med. 2006;57:195-206. doi:10.1146/ annurev.med.57.011205.135654

3. Nickel JC, Tripp DA, Chuai S, et al. Psychosocial variables affect the quality of life of men diagnosed with chronic prostatitis/chronic pelvic pain syndrome. BJU Int. 2008;101:59-64. doi:10.1111/ j.1464-410X.2007.07196.x

4. Krsmanovic A, Tripp DA, Nickel JC, et al. Psychosocial mechanisms of the pain and quality of life relationship for chronic prostatitis/ chronic pelvic pain syndrome (CP/CPPS). Can Urol Assoc J. 2014;8:403-408. doi:10.5489/cuaj.2179

5. Egan KJ, Krieger JN. Psychological problems in chronic prostatitis patients with pain. Clin J Pain. 1994;10:218-226. doi:10.1097/ 00002508-199409000-00008

6. Wang YL, Han QQ, Gong WQ, et al. Microglial activation mediates chronic mild stress-induced depressive- and anxiety-like behavior in adult rats. J Neuroinflamm. 2018;15:21. doi:10.1186/s12974-018-1054-3

7. Reus GZ, Fries GR, Stertz L, et al. The role of inflammation and microglial activation in the pathophysiology of psychiatric disorders. Neuroscience. 2015;300:141-154. doi:10.1016/j.neuroscience.2015.05.018

8. Han Y, Zhang L, Wang Q, et al. Minocycline inhibits microglial activation and alleviates depressive-like behaviors in male adolescent mice subjected to maternal separation. Psychoneuroendocrinology. 2019;107:37-45. doi:10.1016/j.psyneuen.2019.04.021

9. Stence N, Waite M, Dailey ME. Dynamics of microglial activation: a confocal time-lapse analysis in hippocampal slices. Glia. 2001;33:256266. doi:10.1002/1098-1136(200103)33:3<256::aid-glia1024>3.0.co;2-j

10. Graeber MB, Streit WJ. Microglia: biology and pathology. Acta Neuropathol. 2010;119:89-105. doi:10.1007/s00401-009-0622-0

11. Slavich GM, Irwin MR. From stress to inflammation and major depressive disorder: a social signal transduction theory of depression. Psychol Bull. 2014;140:774-815. doi:10.1037/a0035302

12. Hu C, Yang H, Zhao Y, et al. The role of inflammatory cytokines and ERK1/2 signaling in chronic prostatitis/chronic pelvic pain syndrome with related mental health disorders. Sci Rep. 2016;6:28608. doi:10.1038/srep28608 
13. Catena-Dell'Osso M, Bellantuono C, Consoli G, Baroni S, Rotella F, Marazziti D. Inflammatory and neurodegenerative pathways in depression: a new avenue for antidepressant development? Curr Med Chem. 2011;18:245-255. doi:10.2174/ 092986711794088353

14. Hepgul N, Cattaneo A, Zunszain PA, Pariante CM. Depression pathogenesis and treatment: what can we learn from blood mRNA expression? BMC Med. 2013;11:28. doi:10.1186/1741-7015-11-28

15. Corona AW, Norden DM, Skendelas JP, et al. Indoleamine 2,3-dioxygenase inhibition attenuates lipopolysaccharide induced persistent microglial activation and depressive-like complications in fractalkine receptor $(\mathrm{CX}(3) \mathrm{CR} 1)$-deficient mice. Brain Behav Immun. 2013;31:134-142. doi:10.1016/j.bbi.2012.08.008

16. Xu Y, Sheng H, Tang Z, Lu J, Ni X. Inflammation and increased IDO in hippocampus contribute to depression-like behavior induced by estrogen deficiency. Behav Brain Res. 2015;288:71-78. doi:10.1016/ j.bbr.2015.04.017

17. Zhu CB, Blakely RD, Hewlett WA. The proinflammatory cytokines interleukin-1beta and tumor necrosis factor-alpha activate serotonin transporters. Neuropsychopharmacology. 2006;31:2121-2131. doi:10.1038/sj. npp. 1301029

18. Haroon E, Miller AH. Inflammation effects on brain glutamate in depression: mechanistic considerations and treatment implications. Curr Top Behav Neurosci. 2017;31:173-198. doi:10.1007/7854_2016_40

19. He XX, Wei ZK, Zhou ES, et al. Baicalein attenuates inflammatory responses by suppressing TLR4 mediated NF-kappa B and MAPK signaling pathways in LPS-induced mastitis in mice. Int Immunopharmacol. 2015;28:470-476. doi:10.1016/j.intimp.2015.07.012

20. Wang W, Zhou PH, Xu CG, Zhou XJ, Hu W, Zhang J. Baicalein ameliorates renal interstitial fibrosis by inducing myofibroblast apoptosis in vivo and in vitro. BJU Int. 2016;118:145-152. doi:10.1111/ bju. 13219

21. Han ZQ, Zhu SM, Han X, Wang ZA, Wu SW, Zheng RS. Baicalein inhibits hepatocellular carcinoma cells through suppressing the expression of CD24. Int Immunopharmacol. 2015;29:416-422. doi:10.1016/j.intimp.2015.10.021

22. Wei N, Wei YH, Li BR, Pang LL. Baicalein promotes neuronal and behavioral recovery after intracerebral hemorrhage via suppressing apoptosis, oxidative stress and neuroinflammation. Neurochem Res. 2017;42:1345-1353. doi:10.1007/s11064-017-2179-y

23. Zhang CYY, Zeng MJ, Zhou LP, et al. Baicalin exerts neuroprotective effects via inhibiting activation of GSK3 beta/NF-kappa B/NLRP3 signal pathway in a rat model of depression. Int Immunopharmacol. 2018;64:175-182. doi:10.1016/j.intimp.2018.09.001

24. Breser ML, Motrich RD, Sanchez LR, Rivero VE. Chronic pelvic pain development and prostate inflammation in strains of mice with different susceptibility to experimental autoimmune prostatitis. Prostate. 2017;77:94-104. doi:10.1002/pros.23252

25. Rivero VE, Cailleau C, Depiante-Depaoli M, Riera CM, Carnaud C. Non-obese diabetic (NOD) mice are genetically susceptible to experimental autoimmune prostatitis (EAP). J Autoimmun. 1998;11:603610. doi:10.1006/jaut.1998.0248

26. Liu W, Zhai X, Li H, Ji L. Depression-like behaviors in mice subjected to co-treatment of high-fat diet and corticosterone are ameliorated by AICAR and exercise. J Affect Disord. 2014;156:171-177. doi:10.1016/j.jad.2013.11.025
27. Pellow S, Chopin P, File SE, Briley M. Validation of open: closedarm entries in an elevated plus-maze as a measure of anxiety in the rat. $J$ Neurosci Methods. 1985;14:149-167. doi:10.1016/0165-0270(85) 90031-7

28. Porsolt RD, Bertin A, Blavet N, Deniel M, Jalfre M. Immobility induced by forced swimming in rats: effects of agents which modify central catecholamine and serotonin activity. Eur $J$ Pharmacol. 1979;57:201-210. doi:10.1016/0014-2999(79)90366-2

29. Steru L, Chermat R, Thierry B, Simon P. The tail suspension test: a new method for screening antidepressants in mice. Psychopharmacology (Berl). 1985;85:367-370. doi:10.1007/bf00428203

30. Garcia-Cabezas MA, John YJ, Barbas H, Zikopoulos B. Distinction of neurons, glia and endothelial cells in the cerebral cortex: an algorithm based on cytological features. Front Neuroanat. 2016;10:107. doi:10.3389/fnana.2016.00107

31. Zhao Y, Wang Q, Jia M, et al. (+)-Sesamin attenuates chronic unpredictable mild stress-induced depressive-like behaviors and memory deficits via suppression of neuroinflammation. $J$ Nutr Biochem. 2018;64:61-71. doi:10.1016/j.jnutbio.2018.10.006

32. Qiu HM, Yang JX, Jiang XH, et al. Upregulating serotonin transporter expression and downregulating monoamine oxidase-A and indoleamine 2, 3-dioxygenase expression involved in the antidepressant effect of sodium valproate in a rat model. Neuroreport. 2014;25:1338-1343. doi:10.1097/WNR.0000000000000269

33. Zhang YP, Wang HY, Zhang C, et al. Mifepristone attenuates depression-like changes induced by chronic central administration of interleukin-lbeta in rats. Behav Brain Res. 2018;347:436-445. doi:10.1016/j.bbr.2018.03.033

34. Burke NN, Geoghegan E, Kerr DM, Moriarty O, Finn DP, Roche M. Altered neuropathic pain behaviour in a rat model of depression is associated with changes in inflammatory gene expression in the amygdala. Genes Brain Behav. 2013;12:705-713. doi:10.1111/gbb.12080

35. Pan W, Zhang GF, Li HH, et al. Ketamine differentially restores diverse alterations of neuroligins in brain regions in a rat model of neuropathic pain-induced depression. Neuroreport. 2018;29:863-869. doi:10.1097/WNR.0000000000001045

36. Kaur D, Sharma V, Deshmukh R. Activation of microglia and astrocytes: a roadway to neuroinflammation and Alzheimer's disease. Inflammopharmacology. 2019. doi:10.1007/s10787-019-00580-x

37. Ghaemi A, Alizadeh L, Babaei S, et al. Astrocyte-mediated inflammation in cortical spreading depression. Cephalalgia. 2018;38:626638. doi: $10.1177 / 0333102417702132$

38. Takahashi K, Nakagawasai O, Nemoto W, et al. Memantine ameliorates depressive-like behaviors by regulating hippocampal cell proliferation and neuroprotection in olfactory bulbectomized mice. Neuropharmacology. 2018;137:141-155. doi:10.1016/j.neuropharm.2018.04.013

39. Raison CL, Capuron L, Miller AH. Cytokines sing the blues: inflammation and the pathogenesis of depression. Trends Immunol. 2006;27:24-31. doi:10.1016/j.it.2005.11.006

40. Mendonca MS, Mangiavacchi PM, De Sousa PF, et al. Epigenetic variation at the SLC6A4 gene promoter in mother-child pairs with major depressive disorder. J Affect Disord. 2018;245:716-723. doi:10.1016/j.jad.2018.10.369

41. Mayhew J, Beart PM, Walker FR. Astrocyte and microglial control of glutamatergic signalling: a primer on understanding the disruptive role of chronic stress. $J$ Neuroendocrinol. 2015;27:498-506. doi: $10.1111 /$ jne. 12273 


\section{Publish your work in this journal}

Neuropsychiatric Disease and Treatment is an international, peerreviewed journal of clinical therapeutics and pharmacology focusing on concise rapid reporting of clinical or pre-clinical studies on a range of neuropsychiatric and neurological disorders. This journal is indexed on PubMed Central, the 'PsycINFO' database and CAS, and is the official journal of The International Neuropsychiatric Association (INA). The manuscript management system is completely online and includes a very quick and fair peer-review system, which is all easy to use. Visit http://www.dovepress.com/testimonials.php to read real quotes from published authors. 